\title{
Localization and Diffusion of Positive Muons in Metals
}

\author{
By \\ H. Orth ${ }^{a}$, K.-P. Döring ${ }^{b}$, M. Gladisch ${ }^{a}$, \\ D. Herlach ${ }^{b}$, W. Maysenhölder ${ }^{\mathrm{c}}$, H. Metz ${ }^{\mathrm{c}}$, G. zu Putlitz ${ }^{\mathrm{a}}$, \\ A. Seeger ${ }^{b, c}$, J. Vetter ${ }^{a}, W$. Wahl ${ }^{a}$, \\ M. Wigand ${ }^{\mathrm{a}}$ and E. Yagi ${ }^{\mathrm{c}, *}$ \\ " Physikalisches Institut der Universität Heidelberg, Heidelberg, \\ b Institut für Theoretische und Angewandte Physik der Universität Stuttgart, Stuttgart, \\ ' Max-Planck-Institut für Metallforschung, Institut für Physik, Stuttgart
}

(Received December 28, 1978)

Positive muons / Muon spin rotation / Location of muons / Diffusion of muons / Hydrogen in metals

The basic properties of positive muons (positive elementary charge, spin $1 / 2$, mass ratio muon/proton $\approx 1 / 9$, large magnetic moment, parity-non-conserving decay into positron plus neutrini) and their availability in the form of intense spin-polarized beams at "meson factories" permit, by means of the $\mu^{+}$SR ("Muon Spin Rotation") technique, to investigate their interaction with magnetic fields in metals.

In the present paper we shall briefly review the $\mu^{+} \mathrm{SR}$ technique and its application to the study of location and diffusion of muons in metals using the dipolar interaction with the magnetic moments of the nuclei. The general ideas of how the measured relaxation rates are affected by the muon mobility and the trapping and detrapping at impurities are outlined and illustrated by measurements on high-purity single crystals of $\mathrm{Be}, \mathrm{Nb}$, and $\mathrm{Ta}$.

It is shown that a few ppm of impurities may suffice to trap a substantial fraction of the muons during their lifetime, indicating that the muon has a rather high mobility, even at liquid,hydrogen or liquid-helium temperatures. Since the positive muon may be looked upon as a light isotope of the hydrogen nuclides, these results provide interesting information on the quantum effects in hydrogen diffusion.

Die Grundeigenschaften positiver Müonen (positive Elementarladung, Spin 1/2, Massenverhältnis Müon/Proton $\approx 1 / 9$, großes magnetisches Moment, paritätsverletzender Zerfall in ein Positron und zwei Neutrinos) und ihre Verfügbarkeit in Form intensiver spinpolarisierter Strahlen in „Mesonen-Fabriken“ gestatten es, mit Hilfe der $\mu^{+}$SR (,Müon-Spin-Rotation")Methode die Wechselwirkung der Müonen mit Magnetfeldern in Metallen zu studieren.

In dieser Arbeit geben wir einen kurzen Überblick über die $\mu^{+}$SR-Methode und ihre Anwendung auf das Studium der Lokalisierung und Diffusion von Müonen in Metallen für den

* Recipient of an Alexander-von-Humboldt award. 
Fall der dipolaren Wechselwirkung mit den magnetischen Momenten der Kerne. Die Zusammenhänge zwischen den gemessenen Relaxationsraten und der Müonenbeweglichkeit und dem Einfang und Entweichen an Verunreinigungen werden dargestellt und anhand von Messungen an hochreinen $\mathrm{Be}-, \mathrm{Nb}$ - und Ta-Einkristallen veranschaulicht.

Es wird gezeigt, daß wenige ppm Verunreinigungen ausreichen können, um einen beträchtlichen Teil der Müonen während ihrer Lebensdauer einzufangen, was auf eine hohe Beweglichkeit des Müons bis zu den Temperaturen des flüssigen Wasserstoffs oder Heliums hindeutet. Da das positive Müon als ein leichtes Isotop der Wasserstoff-Nuklide betrachtet werden kann, liefern diese Resultate interessante Information über Quanteneffekte in der Wasserstoffdiffusion.

\section{Introduction}

With its positive elementary charge, spin $1 / 2$, and its mass 207 times the electron mass or $1 / 9$ of the proton mass, the positive muon $\left(\mu^{+}\right)$may well be regarded as a light isotope of the proton. Polarized $\mu^{+}$obtained from the decay of pions $\left(\pi^{+}\right)$may be implanted into the bulk of a metal regardless of its hydrogen solubility. Muons decay with a mean life $\tau_{\mu}=2.2 \mu \mathrm{s}$; the emission of relativistic positrons provides information on the depolarization of the muons caused by the interaction of the magnetic moment of the muon (3.18 times that of the proton) with the magnetic fields in the metal. These fields may come from nuclear or electronic magnetic moments, or may be generated by the orbital motion of electrons in superconductors.

The $\mu^{+}$SR ("Muon Spin Rotation") technique employed in the present paper is analogous to the measurement of the transverse relaxation by NMR (Nuclear Magnetic Resonance) but does neither require a large number of spins (during $\mu^{+} \mathrm{SR}$ measurements the sample contains at most one muon at a time), nor radio-frequency irradiation, nor large applied magnetic fields for creating a detectable polarisation ${ }^{1}$.

Under suitable conditions the $\mu^{+}$SR technique is capable of providing fairly direct information on the localization of positive muons in metals. Another important aspect is the study of muon diffusion on a time scale of the order of magnitude of $\tau_{\mu}$. It uses the phenomenon of motional averaging over spatially varying magnetic fields (in NMR known as motional narrowing of the line-width). The range of the muon diffusion coefficients that may be investigated in this way is determined by the strength of the magnetic interaction employed and by the spatial scale of the inhomogeneity of the magnetic field.

The present study makes use of the dipolar interaction between the magnetic moments of the nuclei and those of the muons. In this case the

\footnotetext{
${ }^{1}$ For details of the $\mu^{+}$SR technique the reader is referred to two recent review articles [1,2]. Further information on the basic physical properties of muons may be found in the review by Scheck [3].
} 
inhomogeneity scale is the lattice parameter $a_{0}$ and the order of magnitude of the muon diffusion coefficients that may be determined is $a_{0}^{2} / 10 \tau_{\mu}$. This order of magnitude is very small compared with the hydrogen diffusion coefficients that are usually studied in metals. Hence the muon measurements are carried out at rather low temperatures and quantum effects are likely to be important. This may have two effects on the diffusion process: The big difference in zero-point energy between positive muons and protons results in a different level structure and may even give rise to different diffusion mechanisms [4]. Because of the smaller mass of the muons, for a given mechanism tunneling will affect the diffusion of positive muons up to much higher temperatures than that of protons.

Whereas on the one hand the possibility to study muon location and diffusion at low temperatures permits to investigate quantum effects in considerable detail, it is beset by the complication that small binding energies of the order of magnitude of a few hundredths of an $\mathrm{eV}$ may suffice to trap the muons at impurities for times comparable with $\tau_{\mu}$. By studying the trapping quantitatively one may obtain information on the low-temperature trapping of hydrogen by structural imperfections in metals that would be difficult to obtain otherwise ${ }^{2}$. On the other hand, trapping suppresses motional averaging and hence may let the muon diffusion coefficients appear too low. In the study of muon diffusion by the $\mu^{+} \mathrm{SR}$ technique it is therefore of utmost importance to work with very pure and well characterized specimens.

- These problems may be somewhat alleviated by employing the muon spin relaxation in longitudinal magnetic fields, and we hope to report on the results obtained with this technique in near future.

In the present paper we shall briefly review the $\mu^{+} \mathrm{SR}$ technique in transverse magnetic fields $\mathbf{B}_{\mathrm{appl}}$ and discuss how the measured relaxation rates are affected by the muon mobility and by muon trapping and detrapping at impurities. The general ideas are illustrated by measurements on high-purity single crystals of beryllium, niobium, and tantalum.

\section{Experimental Techniques and Data Analysis}

Spin-polarized $\mu^{+}$obtained from $\pi^{+}$decaying in flight in the superconducting muon channel at Schweizerisches Institut für Nuklearforschung (SIN), Villigen, Switzerland, are stopped in monocrystalline metal targets. The "spin-phase relaxation" in magnetic fields $5 \mathrm{mT}<B_{\text {appl }}<25 \mathrm{mT}$ applied transverse to the muon polarization is determined at temperatures between $2.5 \mathrm{~K}$ and room temperature by detecting the decay positrons (emitted with a probability distribution that is asymmetric with respect to the $\mu^{+}$spins because of the parity non-conservation in Weak Interactions) in

\footnotetext{
${ }^{2}$ The trapping of hydrogen in metals has recently been reviewed by Wert [5].
} 
four scintillation-counter telescopes at $0^{\circ}, 90^{\circ}, 180^{\circ}$, and $270^{\circ}$ with respect to the muon beam, covering together a total solid angle of approximately seven tenth of $4 \pi$. More details concerning the apparatus are given elsewhere [6].

The spectra obtained from the individual telescopes are analyzed in terms of

$$
\mathrm{d} N^{(j)}(t)=N^{(j)} \exp \left(-t / \tau_{\mu}\right) \int_{\Omega^{(j)}}\left[1+A^{(j)} R(t) \cos \left(\omega_{0} t-\phi^{(j)}\right)\right] \mathrm{d} \Omega^{(j)} \mathrm{d} t+B^{(j)},
$$

where $N^{(j)}(t)$ denotes the number of counts in counter $j$ as a function of the time $t$ since the entry of the muons into the sample, $R(t)$ the relaxation function, and $\omega_{0}=\gamma_{\mu} B_{\text {appl }}$ the spin precession frequency of the $\mu^{+}$in the applied field. Furthermore, $\phi^{(j)}$ are the angles between the directions of the initial $\mu^{+}$polarization and the $j$-th counter telescope (which covers the solid angle $\left.\Omega^{(i)}\right), A^{(i)}$ the asymmetries at $t=0, B^{(i)}$ the random-coincidence backgrounds, and $N_{0}^{(j)}$ normalization factors.

Since in the present work the depolarization within $\tau_{\mu}$ is relatively small, the time-dependent precession amplitudes $A^{(j)} R(t)$ are approximated by a step-function assuming the values $A_{i}^{(j)}$ during successive time-intervals $t_{i}<t$ $\leq t_{i}+2 \pi / \omega_{0}$. By comparing the amplitudes $\bar{A}_{i}$ obtained by averaging over the four counters with calculations based on specific models one may obtain physical quantities pertaining to muon diffusion or location. A widely used measure of the relaxation rate is the reciprocal of the time interval $t_{e}$ within which the precession amplitude drops by a factor $1 / e$. This parameter may be obtained model-independently provided the amplitude decreases so fast that $t_{e}$ falls within the observed time interval of typically $7 \cdot 10^{-6} \mathrm{~s}$, as is the case in our $\mathrm{Nb}$ measurements below $60 \mathrm{~K}$.

\section{Relaxation Functions and Relaxation Rates}

In the present experiments we have to take into account the dipolar interactions between the magnetic moments of the muons and the nuclei, and the quadrupolar interactions between the nuclear quadrupole moments $Q$ and the electrical field gradient $\nabla \nabla \mathrm{V}$ generated by the muons at the nuclear sites. In several limiting cases $R(t)$ is known from NMR theory.

(i) The $\mu^{+}$are thermalized in times short compared to their lifetime and form localized states at specific sites in the crystal lattice. To a good approximation, the relaxation function is then given by a Gaussian [7]

$$
R(t)=\exp \left(-\Gamma_{0}^{2} t^{2}\right) \equiv \exp \left[-\left(t / t_{e}\right)^{2}\right]
$$

with

$$
\Gamma_{0}^{2}=\frac{\hbar^{2}}{6} \mathrm{I}(\mathrm{I}+1) \gamma_{\mathrm{I}}^{2} \gamma_{\mu}^{2}\left(\frac{\mu_{0}}{4 \pi}\right)^{2} \sum_{j} \frac{\left(1-3 \cos ^{2} \theta_{j}\right)^{2}}{r_{j}^{6}}
$$


where I denotes the nuclear spin, $\gamma_{\mathrm{I}}$ and $\gamma_{\mu}$ the gyromagnetic ratios of the nuclei and the muon, $r_{j}$ the length of the vector $\mathbf{r}_{j}$ connecting the $\mu^{+}$and the $j$-th nucleus, and $\theta_{j}$ the angle between $\mathbf{B}_{\text {appl }}$ and $\mathbf{r}_{j}$, the sum extending over all nuclei in the crystal. Equation(3) is only valid if the magnetic interaction energy $\hbar \omega_{1}$ of the nuclear magnetic moments with the applied field,

$$
\hbar \omega_{1}=\hbar \gamma_{1} B_{\mathrm{appl}},
$$

exceeds considerably the interaction energy $\hbar \omega_{Q}$ of the nuclear quadrupole moment $Q$ with the electric field gradient $V_{z z}$ generated by the muon [8]:

$$
\hbar \omega_{Q}=e Q V_{z z}(1+\gamma) / 4 \mathrm{I}(2 \mathrm{I}-1) .
$$

Here, $\gamma$ is an enhancement factor taking into account the electron polarization by the electric field ( $\gamma$ is known as Sternheimer factor or as Bloch-wave enhancement factor). For $\hbar \omega_{1}<\hbar \omega_{Q}$ the dependence of $\Gamma_{0}$ on the crystallographic direction of $\mathbf{B}_{\mathrm{appl}}$ is considerably reduced since then the quantization axis of the nuclear spins is no longer determined by the applied field alone. In this case the relaxation rate has to be calculated numerically from appropriate models for $\nabla \nabla \mathrm{V}$ and $\gamma$ (see, e.g. [9]).

(ii) The muons diffuse so fast that each individual $\mu^{+}$averages over the magnetic field distribution within its lifetime. Then the distribution of the precession frequencies around the frequency $\omega_{0}$ corresponding to the applied field will be very narrow and the depolarization very small (known as "motional narrowing" in NMR).

(iii) The motion of the $\mu^{+}$is described by discrete jumps between equivalent sites in the crystal. A often-used approximation which does give the limiting cases (i) and (ii) correctly is [10]

$$
P(t)=\exp \left\{-2 \Gamma_{0}^{2} \tau_{c}^{2}\left[\exp \left(-\frac{t}{\tau_{c}}\right)-1+\frac{t}{\tau_{c}}\right]\right\},
$$

where $\Gamma_{0}$ is given by (3) and the correlation time $\tau_{c}$ is related to the mean time of residence $\bar{\tau}$ of a $\mu^{+}$at a given site and hence to the diffusion coefficient $D^{\mu^{+}}$of the muon. In particular, for jumps between octahedral or tetrahedral interstitial sites in a body-centred cubic lattice one finds

$$
D^{\mu^{+}}=a_{0}^{2} / 36 \tau_{c}
$$

for octahedral and

$$
D^{\mu^{+}}=a_{0}^{2} / 72 \tau_{c}
$$

for tetrahedral occupancy, taking $\tau_{c}=\frac{2}{3} \bar{\tau}[2]$.

In general - taking into account the possibility of trapping by impurities or defects and allowing for thermally activated detrapping, or considering complicated diffusion paths involving non-equivalent interstitial sites - the relaxation function cannot be given analytically. In these cases a quantitative analysis of the data will have to rely on numerical calculations for $R(t)$ based on detailed diffusion and trapping models. 

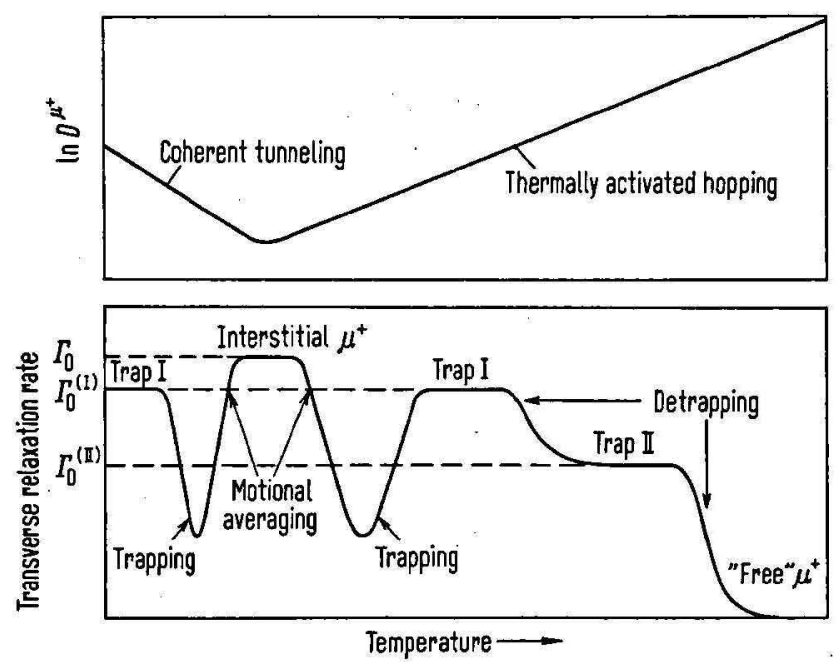

Fig. 1. Qualitative temperature dependence of the transverse relaxation rate (bottom) for a model showing a minimum in the temperature variation of the muon diffusion coefficient (top). The possible effects of two types of traps are indicated

In the following we discuss qualitatively a fairly general model which should provide a good frame-work for the interpretation of the experiments. It is assumed that $D^{\mu^{+}}$shows a minimum as a function of temperature (Fig. 1). Such a temperature dependence arises if at high temperatures the muon diffusivity is due to thermally activated hopping, whereas at low temperatures the muon propagates by "coherent tunneling" with its mean free path limited by phonon scattering. In Fig. 1 it has been assumed that near the minimum $D^{\mu^{+}}$is sufficiently small for a muon to stay essentially at one interstitial site (characterized by the relaxation rate $\Gamma_{0}$ ) during its lifetime. At both lower and higher temperatures motional averaging sets in. If the increase in $D^{\mu^{+}}$is large enough to let the majority of the muons reach traps (e.g., type-I solute atoms) the relaxation rate may rise again to the level $\Gamma_{0}{ }^{(1)}$ characteristic for these traps. This requires (a) the product of the capture rate $\sigma^{(\mathbf{l})}$ and the concentration $C^{(\mathbf{l})}$ of these traps to be larger than the sum of the corresponding products for all other impurities and (b) the binding energy between muons and traps to be large enough to prevent the muons from escaping during their lifetime. The latter condition will be violated with increasing temperature, since thermal activation enhances the escape rate. During its lifetime a muon may visit many traps, and another phenomenon of 'motional averaging', accompanied by a decrease of the relaxation rate, may occur. In Fig. 1 we have assumed that a second type of trap exists with $\sigma^{(\text {II) }} C^{(\text {II) }} \ll \sigma^{(\text {(I) }} C^{(\text {I) }}$ but with a substantially higher binding energy than the type-I traps. Then the relaxation rate as a function of temperature may show 
another plateau at the level $\Gamma_{0}^{(\text {II) }}$ corresponding to muons immobilized during most of their lifetime at type-II traps. At sufficiently high temperatures trapping will become unimportant and the relaxation rate will tend to zero [case (ii) above].

\section{Sample Preparation ${ }^{3}$}

\section{Beryllium}

A single crystal $25 \mathrm{~mm}$ in diameter and $12 \mathrm{~mm}$ thick was spark-cut from a rod which had been vacuum melted from high-purity material and subsequently zone refined 10 times. The residual resistivity ratio of the sample $\Gamma$ $\equiv R_{293 \mathrm{~K}} / R_{4.2 \mathrm{~K}}=400$ corresponds to a purity of $99.993 \mathrm{wt}$. $\% \mathrm{Be}$. The remaining $70 \mathrm{ppm}$ impurities are mainly iron [11].

\section{Tantalum}

From a monocrystalline rod $4 \mathrm{~mm}$ in diameter (with a $\langle 111\rangle$-direction along the axis) grown by floating-zone melting in ultra-high vacuum using electron bombardment [12] seven crystals were cut $20 \mathrm{~mm}$ long each. They were arranged in the cryostat with their axes parallel to $\mathbf{B}_{\text {appl }}$ with an exposure of $\sim 7 \mathrm{~g} / \mathrm{cm}^{2} \mathrm{Ta}$ to the $\mu^{+}$beam. The residual resistivity ratio was $\Gamma=5000$. The overall concentration of interstitial impurities was $\mathrm{C}+\mathrm{O}+\mathrm{N}<5$ at.ppm.

\section{Niobium}

This target consisted of three rods $9 \mathrm{~mm}$ in diameter and $25 \mathrm{~mm}$ long cut from a single crystal (grown in the same way as the Ta crystals) with a $\langle 111\rangle$ direction forming an angle of $21^{\circ}$ with the axis. Here $\Gamma=3000, \mathrm{~N}$ $\approx 2$ at. ppm, $\mathrm{O}<1$ at. ppm, and $\mathrm{Ta} \approx 5$ at. ppm.

\section{Results and Discussion}

\subsection{Beryllium}

In Fig. 2 the temperature dependence of the relaxation rate [obtained by fitting (6) to the spectra] is shown for an external magnetic field $B_{\text {appl }}$ $=24.6 \mathrm{mT}$ forming an angle of $50^{\circ}$ with the hexagonal axis of the crystal.

${ }^{3}$ The Be crystal was prepared at Max-Planck-Institut für Metallforschung, Institut für Werkstoffwissenschaften, Pulvermetallurgisches Laboratorium, Stuttgart (F. Aldinger, E. Bruckner), and the $\mathrm{Ta}$ and $\mathrm{Nb}$ crystals were grown at Max-Planck-Institut für Metallforschung, Institut für Physik, Stuttgart (H. Schultz, R. Henes, P. Keppler). 


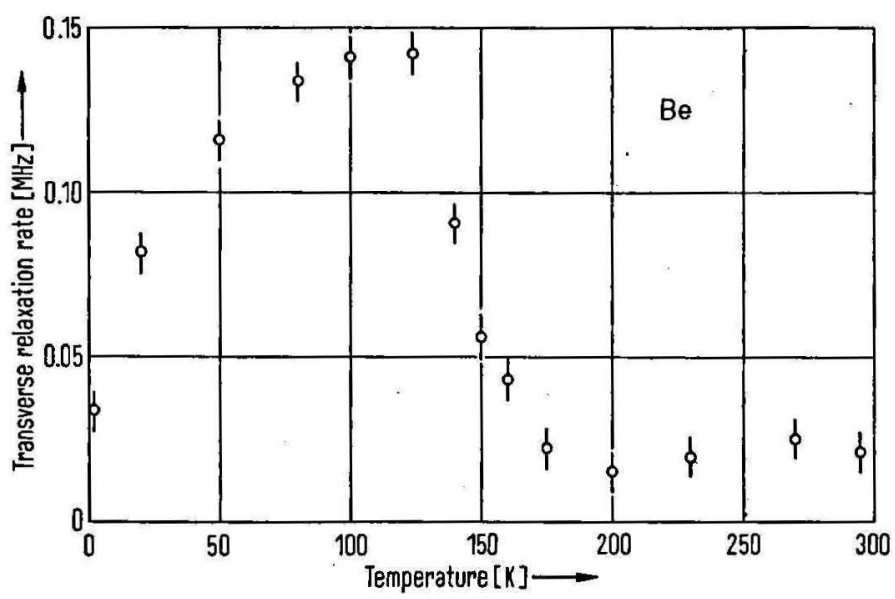

Fig. 2. Transverse relaxation rates of $\mu^{+}$in beryllium with $B_{\text {appl }}=24.6 \mathrm{mT}$ forming an angle of $50^{\circ}$ with the $c$-axis of the crystal. The relaxation rates were obtained by fitting (6) with $\Gamma_{0}$ $=0.14 \mathrm{MHz}$ to the experimental relaxation functions

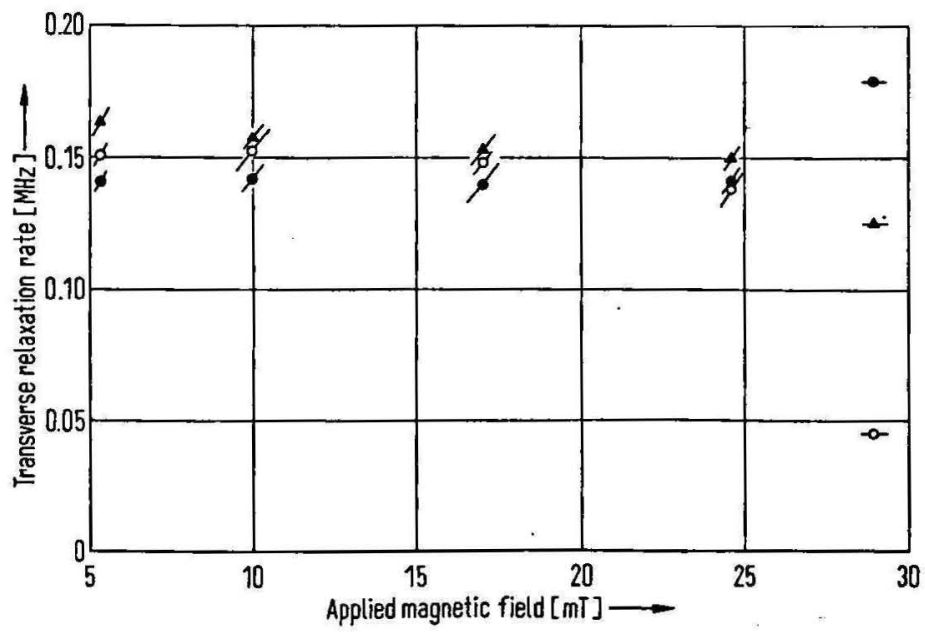

Fig. 3. Orientation and field dependence of the relaxation rate of $\mu^{+}$in Be. The angles between $\mathbf{B}_{\text {appl }}$ and the hexagonal axis are $0^{\circ}(0), 50^{\circ}(\bullet)$, and $90^{\circ}(\boldsymbol{\Lambda})$. On the right-hand side the high-field limits [computed from (3) for muons at octahedral interstitial sites in an otherwise perfect environment] are indicated

Measurements with $\mathbf{B}_{\text {appl }}$ parallel or perpendicular to the $c$-axis gave no significant orientation dependence over the entire temperature range investigated. As is shown in Fig. 3 the dependence of the relaxation rate at $100 \mathrm{~K}$ on the applied magnetic field $B_{\text {appl }}$ is also very weak in the field range $5 \mathrm{mT}$ to $25 \mathrm{mT}$. 
There are basically two possibilities for the interpretation of the preceding facts :

(i) The broad maximum in the relaxation rate at $100 \mathrm{~K}$ is due to the immobilization of the $\mu^{+}$at interstitial sites in an otherwise perfect environment. The weak field and orientation dependence then indicates that the quadrupolar interaction energy $\hbar \omega_{Q}$ is at least of the order of the dipolar interaction energy $\hbar \omega_{1}$ and hence the relaxation rate at $100 \mathrm{~K}$ may not be computed from (3). From self-consistent calculations of the potential seen by a positive muon in $\mathrm{Be}$ we conclude that the $\mu^{+}$cannot be localized at tetrahedral interstices. Model calculations of the relaxation rates for muons trapped at trigonal or octahedral sites (taking into account the interaction of the electric-field gradient created by the $\mu^{+}$with the quadrupole moment of the $\mathrm{Be}$ nuclei) further rule out the trigonal site but are compatible with octahedral occupancy with $\sim 10 \%$ outward displacement of the nearest neighbours.

Under these assumptions, the decrease of the relaxation rate above $120 \mathrm{~K}$ is attributed to thermally activated hopping with an activation energy of roughly $100 \mathrm{meV}$ and a preexponential factor $\tau_{c}(T \rightarrow \infty)$ of the order $10^{-10} \mathrm{~s}$ [13]. The low-temperature decrease of the relaxation rate has then to be explained by an increase in $D^{\mu^{+}}$due to coherent tunneling.

(ii) The maximum at $100 \mathrm{~K}$ is due to trapping of the muons by impurities. In this case a binding energy for the muon-impurity complex of the order of $0.1 \mathrm{eV}$ is estimated from the decrease of $t_{e}^{-1}$ above $120 \mathrm{~K}$. Information on the temperature dependence of $D^{\mu^{+}}$may be obtained by comparing the relaxation rate below $100 \mathrm{~K}$ with appropriate model calculations for $R(t)$.

The data on hydrogen diffusion in Be are scarce. Jones and Gibson [14] obtained somewhat indirectly for the diffusion coefficient of tritium

$$
D^{\mathrm{T}}=3 \cdot 10^{-11} \exp (-0.19 \mathrm{eV} / k T) \mathrm{m}^{2} \mathrm{~s}^{-1} \text {. }
$$

The small values of the activation energy and of the pre-exponential factor suggest that quantum effects may play a rôle up to $10^{3} \mathrm{~K}$ or more. It would therefore be very interesting to have reliable data on $D^{\mu^{+}}$. However, on the basis of the present experimental evidence we cannot decide between the two interpretations outlined above. Further experiments, e.g. in longitudinal fields or on samples of higher purity, are necessary.

\subsection{Tantalum}

The quadrupole moment of ${ }^{181} \mathrm{Ta}$ is exceptionally high $(Q=3.0$ $\left.\cdot 10^{-28} \mathrm{~m}^{2}\right)$. It is therefore likely that for the magnetic field used in our experiments $\left(B_{\mathrm{app} 1}=24 \mathrm{mT}\right)$ we are in the regime $\omega_{1} / \omega_{Q}<1$ at least for the nearest and next-nearest neighbours of the muons ${ }^{4}$. In Fig. 4 we show the

${ }^{4}$ This viewpoint is strongly supported by measurements of Schilling et al. [15], who found no significant dependence of the relaxation rate on $B_{\text {appl }}$ up to $0.6 \mathrm{~T}$. 


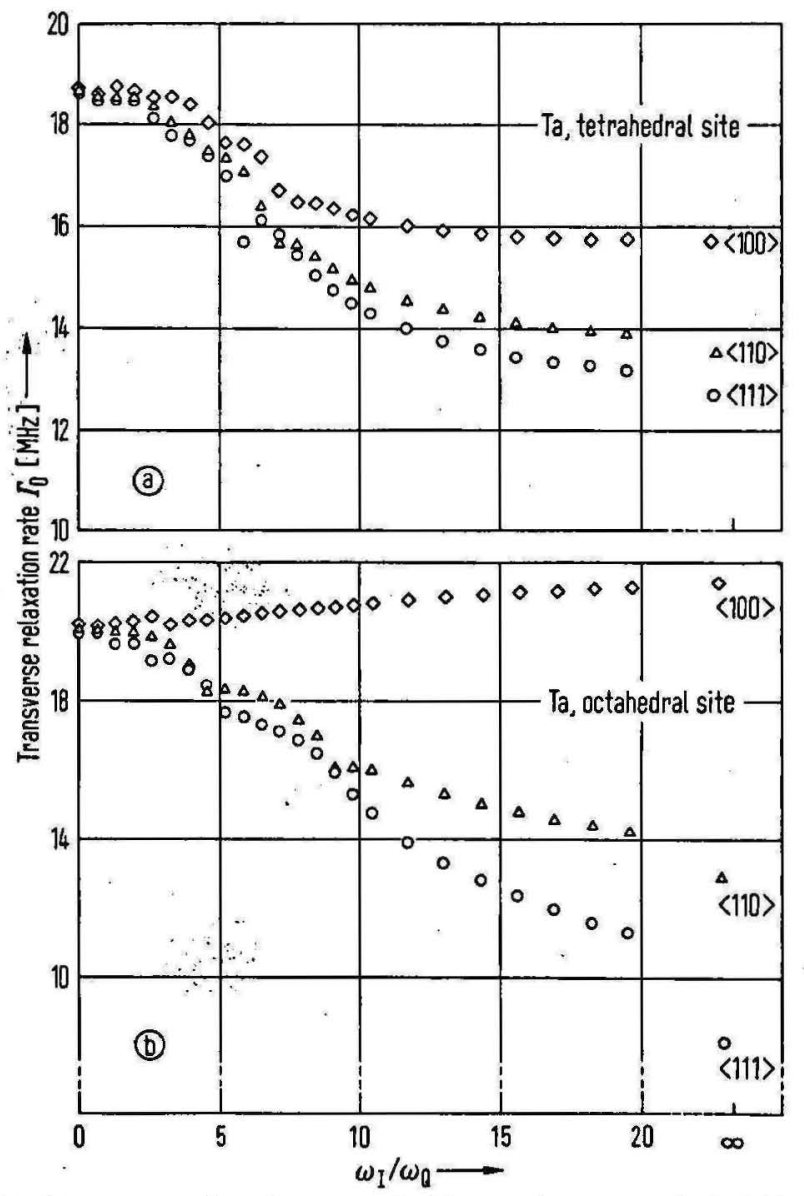

Fig. 4. Calculated transverse relaxation rates of $\mu^{+}$in tantalum at tetrahedral (a) or octahedral (b) interstitial sites as a function of the parameter $\omega_{1} / \omega_{Q}$ (see Sect. 3). The crystallographic orientations of $\mathbf{B}_{\mathrm{appl}}$ are indicated on the right. The relaxation rates given at $\omega_{1} / \omega_{Q}=\infty$ correspond to the limiting cases of zero electric field gradients or large applied fields. Possible displacements of the nuclei by the muon are disregarded. The ordinate scale should be reduced by a factor 100

relaxation rates calculated for point-like muons on tetrahedral (a) or octahedral (b) sites as a function of $\omega_{1} / \omega_{Q}$, where $\omega_{Q}$ pertains to a distance $a_{0} / 2$ from the muon sites. It has been assumed that the electric field gradient has rotational symmetry around the axes nuclei-muons and that it decreases as $r^{-3}$ with the distance $r$ from the muons. For $\mathbf{B}_{\text {appl }}$ parallel to $\langle 100\rangle$ or $\langle 110\rangle$ the relaxation rate is given by $\Gamma_{0}^{2}=\frac{1}{3} \Gamma_{1}^{2}+\frac{2}{3} \Gamma_{\mathrm{II}}^{2}$, where $\Gamma_{\mathrm{I}}$ and $\Gamma_{\mathrm{II}}$ represent the relaxation rates corresponding to nonequivalent sites, subscript II referring to the sites whose tetragonality axes are perpendicular to $\boldsymbol{B}_{\mathrm{appl}}$. The effects of the displacement of the nuclei by the muon have been 


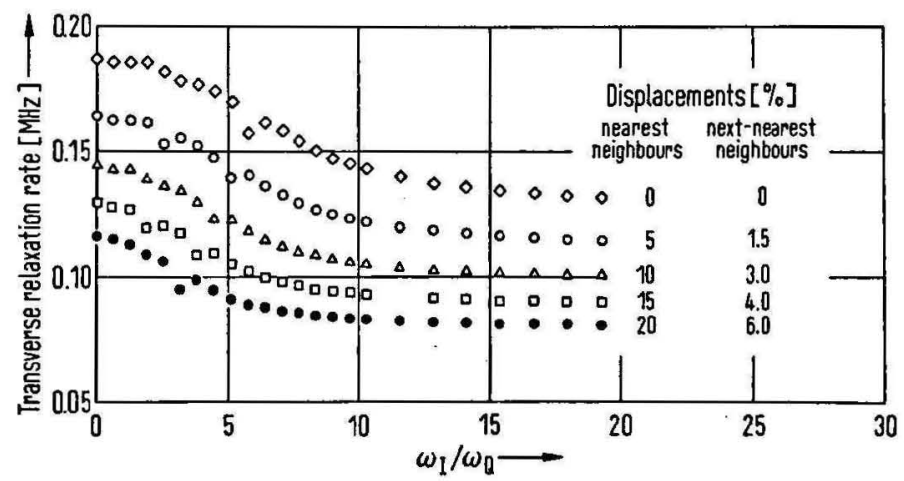

Fig. 5. Effect of displacements of the nearest and next-nearest neighbour nuclei of the $\mu^{+}$at tetrahedral sites in Ta on the calculated relaxation rates for $B_{\text {appl }} \mid l\langle 111\rangle$. The displacements are given in percent of the muon-nucleus separation in an undisturbed lattice and point radially away from the muon

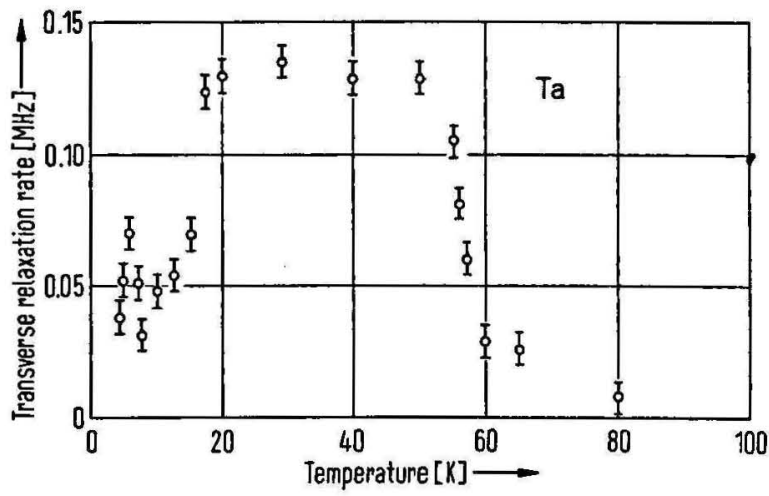

Fig. 6. Experimental relaxation rates of $\mu^{+}$in Ta with $B_{\text {appl }}=24 \mathrm{mT}$ parallel to a $\langle 111\rangle$ direction of the crystal [obtained by fitting (6) with $\Gamma_{0}=0.103 \mathrm{MHz}$ to the measured relaxation functions]

disregarded in Fig. 4. A feeling for the magnitude of these effects may be obtained from Fig. 5, where results for different displacements are given for tetrahedral occupancy and $\mathbf{B}_{\text {appl }} \|\langle 111\rangle^{5}$.

The measured relaxation rates with $\mathbf{B}_{\text {appl }} \|\langle 111\rangle$ (Fig. 6) show a distinct plateau at $t_{e}^{-1} \approx 0.1 \mathrm{MHz}$ extending from $20 \mathrm{~K}$ to $50 \mathrm{~K}$. Neither for tetrahedral nor octahedral occupancy is this value compatible with the above results and reasonable displacements of the nuclei or a reasonable spreadingout of the muon wave-function. A tentative explanation is that muons are

${ }^{5}$ The calculations shown in Figs. 4 and 5 take into account the interaction with all nuclei within a sphere of radius $3 a_{0}$ around the muon. 


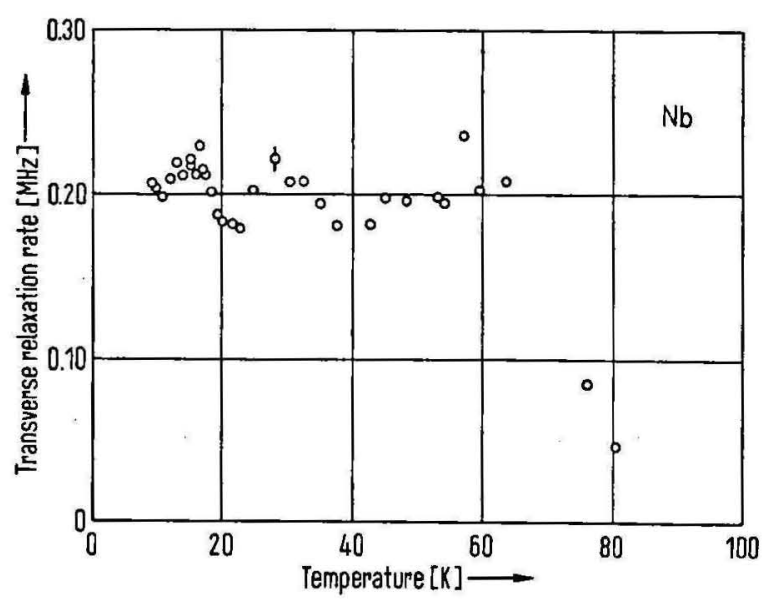

Fig. 7. Experimental relaxation rates of $\mu^{+}$in $\mathrm{Nb}, B_{\text {appl }}=24 \mathrm{mT}$ forming an angle of $21^{\circ}$ with a 〈111) direction of the crystal. Up to $65 \mathrm{~K}$ the relaxation rates $t_{e}^{-1}$ were determined modelindependently; above $65 \mathrm{~K}$ (6) was used with $\Gamma_{0}=0.2 \mathrm{MHz}$

trapped at impurities such as $\mathrm{O}, \mathrm{N}$, or C located on octahedral sites, and that they tunnel in (100) planes between four equivalent sites that are close to the four tetrahedral sites adjacent to the foreign interstitial atom ${ }^{6}$. This interpretation, which is a modification of a proposal made by Buchholz, Völkl and Alefeld [16] and also by Birnbaum and Flynn [17] for hydrogen in $\mathrm{Nb}$, has to be tested by further calculations and control experiments.

\subsection{Niobium}

The maxima and minima of the relaxation rate shown in Fig. 7 are well beyond the scatter of the measurements. According to the discussion of Sect. 3 their existence indicates that trapping and detrapping at impurities must have taken place, and that at least two different types of traps have been involved. An implication of this interpretation is that in the entire temperature range investigated $D^{\mu^{+}}$is so high that within their lifetime a substantial fraction of the muons can reach impurities present in concentrations of a few $\mathrm{ppm}$ only. This conclusion has very recently been confirmed on $\mathrm{Nb}$ with even higher purity (O. Hartmann et al., this conference) which shows relaxation rates $t_{e}^{-1}<0.1 \mathrm{MHz}$ from $3-300 \mathrm{~K}$, indicating strong motional averaging [comp. case (ii) of Sect. 3]. The quantitative determination of $D^{\mu^{+}}$in $\mathrm{Nb}$ remains a task for the future.

${ }^{6}$ By means of blocking experiments Carstanjen and Dünstl (see H.-D. Carstanjen, this conference) have shown that in $\mathrm{Nb}$ deuterons are trapped by nitrogen in positions that are compatible with the above proposal. 


\section{Summary and Conclusions}

The aim of the present paper was to outline the basic ideas of the positivemuon spin rotation method $\left(\mu^{+} \mathrm{SR}\right)$ and its applications to the study of location and diffusion of positive muons in metals. The positive muon may be looked upon as a light hydrogen isotope (mass ratio proton-muon 9:1). $\mu^{+}$SR studies may be performed at low temperatures, where quantum effects are likely to be important and where diffusion experiments on hydrogen are usually difficult or even impossible. Hence such studies are of considerable interest for a deeper understanding of the properties of hydrogen in metals. The general ideas and techniques have been illustrated by selected results on $\mathrm{Be}, \mathrm{Ta}$ and $\mathrm{Nb}$. Lack of space did not permit to give a complete survey of the experimental information available. (The results obtained up to about end of 1977 have been summarized in [2]; more recent experiments may be found in the proceedings of the September 1978 Rorschach conference ${ }^{7}$.)

The experiments have demonstrated the great potential of the method, but few quantitative results have been obtained to-date. This is due to the fact that even small binding energies between muons and foreign atoms (of the order $0.1 \mathrm{eV}$ or less) may suffice to trap the muons in the time and temperature regime to which the experiments pertain and that most of the early work paid little attention to questions of purity and sample characterization. An important conclusion from the experiments carried out so far is that in quite a few of the metals to which the $\mu^{+} \mathrm{SR}$ method is applicable the muon diffusivities $D^{\mu^{+}}$are large compared to $a_{0}^{2} / 10 \tau_{\mu} \approx 5 \cdot 10^{-15} \mathrm{~m}^{2} \mathrm{~s}^{-1}$ at liquid-hydrogen and liquid-helium temperatures. Such large diffusivities allow positive muons to be used as probes for imperfections in metals (including intrinsic defects such as vacancies or small clusters of selfinterstitials [6]) even when their concentration is quite low.

\section{Acknowledgments}

The authors are indebted to Prof. H. Schultz, Dr. F. Aldinger, Dr. K. Schulze, E. Bruckner, R. Henes, and P. Keppler for the preparation and characterization of the samples, to Dr. K. Maier for his advice in the design of the apparatus, and to M. Krenke for his technical assistance. They are also grateful to Prof. $\mathrm{H}$. Teichler for many discussions on problems of muon diffusion and localization in crystals. The authors gratefully acknowledge the support of the Bundesministerium für Forschung und Technologie, Bonn, through their programs "Mittelenergiephysik" and "Nukleare Festkörperforschung", and the cooperation of Schweizerisches Institut für Nuklearforschung (SIN), Villigen.

\section{References}

1. A. Schenck, in Nuclear and Particle Physics at Intermediate Energies (ed. by J. B. Warren), Plenum Press, New York 1976, p. 159.

2. A. Seeger, in Hydrogen in Metals, ed. by G. Alefeld and J. Völkl (Topies in Applied Physics, Vol. 28). Springer, Berlin, Heidelberg, New York 1978, p. 349.

${ }^{7}$ Hyperfine Interactions 6, ns. $1-4$ (1979). 
3. F. Scheck, Phys. Reports 44 (1978) 187.

4. H. Teichler, Proc. 1st Int. Topical Meeting on Muon Spin Rotation, Sept. 4-7, Rorschach/Switzerland, Hyperfine Interactions 6 (1979) 251.

5. Ch. A. Wert, in Hydrogen in Metals, ed. by G. Alefeld and J. Völkl (Topics in Applied Physics, Vol. 29), Springer, Berlin, Heidelberg, New York 1978, p. 305.

6. K. Dorenburg, M. Gladisch, D. Herlach, W. Mansel, H. Metz, H. Orth, G. zu Putlitz, A. Seeger, W. Wahl and M. Wigand, Z. Phys. B 31 (1978) 165.

7. J. H. van Vleck, Phys. Rev. 74 (1948) 1168.

8. O. Hartmann, Phys. Rev. Lett. 39 (1977) 832.

9. P. Jena, S. G. Das and K. S. Singwi, Phys. Rev. Lett. 40 (1978) 264.

10. A. Abragam, The Principles of Nuclear Magnetism, Oxford University Press, London 1961.

11. F. Aldinger, Flow and Fracture of Single Crystals, a chapter in: Beryllium, Science and Technology (D. Webster and G. London, eds.), Plenum Publ. Corp., New York (in press).

12. R. Lachenmann and H. Schultz, Z. Metallkunde 66 (1975) 443.

13. H. Metz, H. Orth, G. zu Putlitz, A. Seeger, H. Teichler, J. Vetter, W. Wahl, M. Wigand, K. Dorenburg, M. Gladisch and D. Herlach, Proc. 1st Int. Topical Meeting on Muon Spin Rotation, Sept. 4-7, Rorschach Switzerland, Hyperfine Interactions 6 (1979) 271.

14. P. M. S. Jones and R. Gibson, Hydrogen in Beryllium Research Report AWRE No. 0-2/67, United Kingdom Atomic Energy Authority, U.K. (February 1967) DMIC No. 69335.

15. H. Schilling, M. Camani, F. N. Gygax, W. Rüegg and A. Schenck, Phys. Lett. 67 A (1978) 231.

16. J. Buchholz, J. Völkl and G. Alefeld, Phys. Rev. Lett. 30 (1973) 318.

17. H. K. Birnbaum and C. P. Flynn, Phys. Rev. Lett. 37 (1976) 25. 